\title{
Phosphorylated retinoblastoma is a biomarker of overall disease control and better progression-free survival of recurrent epithelial ovarian cancer patients treated with second-line topoisomerase inhibitor CPT-11 chemotherapy
}

\author{
Kui Jiang", Guangyan Gao", Lulu Yao \\ Department of Medical Oncology, The Second Affiliated Hospital of Dalian Medical University, Dalian 116027, China \\ Contributions: (I) Conception and design: All authors; (II) Administrative support: L Yao; (III) Provision of study materials or patients: K Jiang; (IV) \\ Collection and assembly of data: All authors; (V) Data analysis and interpretation: L Yao; (VI) Manuscript writing: All authors; (VII) Final approval of \\ manuscript: All authors. \\ \#These authors contributed equally to this work. \\ Correspondence to: Lulu Yao. Department of Medical Oncology, The Second Affiliated Hospital of Dalian Medical University, Dalian 116027, China. \\ Email: yaoluludy2y@163.com.
}

Background: To determine the association of phosphorylated retinoblastoma ( $\mathrm{p}-\mathrm{RB})$ with the clinical outcome of recurrent epithelial ovarian cancer (EOC) patients after CPT-11 chemotherapy.

Methods: We enrolled 27 recurrent EOC patients who had failed first-line chemotherapy and underwent second-line and above CPT-11 chemotherapy from January 2002 to September 2016 in our hospital. The complete medical records of the patients were retrospectively reviewed. The phosphorylation of RB at Ser780 (p-RB) in surgical tumors and 27 normal ovarian tissues was assessed by immunohistochemistry.

Results: The positive rate of $\mathrm{p}-\mathrm{RB}$ expression in EOC tissues was significantly higher than that in normal ovarian tissues $(62.96 \%$ vs. $22.22 \%, \mathrm{P}<0.05)$. p-RB expression had no obvious relationship with the patient's lymph node metastasis, platinum sensitivity, Federation of Gynecology and Obstetrics (FIGO) stage, histological grade, initial symptoms of ascites, pathological type, age, neoadjuvant chemotherapy, or type of first-line chemotherapy (all $\mathrm{P}>0.05$ ). In addition, $\mathrm{p}-\mathrm{RB}$ expression was significantly associated with disease control rates of CPT-11 chemotherapy $(\mathrm{P}<0.001)$. Univariate analysis showed that $\mathrm{p}-\mathrm{RB}$ expression, FIGO stage, lymph node metastasis, and initial symptoms of ascites were factors affecting progression-free survival (PFS) after CPT-11 chemotherapy in recurrent EOC patients (all $\mathrm{P}<0.05)$. Multivariate analysis showed that $\mathrm{p}-\mathrm{RB}$ expression was an independent prognostic factor for PFS in the recurrent EOC patients who accepted CPT-11 chemotherapy $(\mathrm{P}<0.05)$.

Conclusions: The positive rate of p-RB expression in EOC tissues was significantly higher than that in normal ovarian tissues, and p-RB expression was significantly correlated with disease control rates and PFS of recurrent EOC patients after CPT-11 chemotherapy. Our findings suggest that p-RB is a prognostic biomarker for recurrent EOC patients who accept CPT-11 therapy after first-line chemotherapy failure.

Keywords: Retinoblastoma phosphorylation; recurrent epithelial ovarian cancer (recurrent EOC); CTP-11; progression-free survival; disease control rate

Submitted Sep 17, 2018. Accepted for publication Dec 03, 2018.

doi: $10.21037 /$ tcr.2018.12.19

View this article at: http://dx.doi.org/10.21037/tcr.2018.12.19 


\section{Introduction}

It has been estimated that approximately 22,240 new cases of ovarian cancer will be diagnosed and 14,070 women will die of ovarian cancer in the United States in 2018 (1). Due to the special anatomy of the ovary and the lack of appropriate early screening, more than $80 \%$ of patients are diagnosed with advanced ovarian cancer, which also has a high risk of recurrence (2). Chemotherapy is currently one of the major treatments for ovarian cancer (3). However, more than $80 \%$ of patients require second-line treatment due to the emergence of drug-resistance, and there is a significant reduction in second-line chemosensitivity compared with first-line chemotherapy (4). The current second-line and above chemotherapeutic drugs include doxorubicin liposomes, gemcitabine, topotecan, etoposide, ifosfamide, docetaxel, and oxaliplatin. It has been reported that the topoisomerase inhibitor CPT-11 has a similar chemotherapeutic drug efficacy to the above-mentioned drugs but displays no cross-resistance (5). Therefore, in clinical practice, CPT-11 is often chosen as the secondline or third-line chemotherapeutic drug for epithelial ovarian cancer (EOC) because of the high price and unavailability of other second-line drugs such as topotecan. The median overall survival after second-line chemotherapy for recurrent EOC is about 1 year, and the drug-related progression-free survival (PFS) is associated with the overall survival (6). Therefore, in order to improve survival and to achieve individualized treatment, it is particularly important to identify effective biomarkers of CPT-11 efficacy for the improvement of second-line treatment of ovarian cancer.

CPT-11 is a cell-cycle-specific chemotherapeutic drug that disrupts chromatin metabolism in S-phase cells to induce cell death (7). It is well known that one of the key points in regulating cell cycle progression is the G1/S transition (8). The phosphorylation status of retinoblastoma $(\mathrm{RB})$ protein is an important step in controlling this checkpoint (9). In malignant tumor cells, uncontrolled RB phosphorylation results in the constitutive inactivation of RB protein, leading to loss of the G1-phase checkpoint and premature S-phase entry. Thus, the level of phosphorylated $\mathrm{RB}(\mathrm{p}-\mathrm{RB})$ may affect the efficacy of CPT-11 in treating solid tumors (7-9). It has been shown that the proliferation rate of colorectal cancer cells and the $\mathrm{RB}$ protein phosphorylation at Ser780 are associated with poor clinical outcomes of colorectal cancer patients (10); however, whether RB protein phosphorylation at Ser780 can be used as a biomarker for evaluating the effect and prognosis of CPT-11 chemotherapy as a second-line treatment for recurrent ovarian cancer remains to be determined.

In this study, in order to provide the basis for optimizing individualized treatment strategies after the failure of first-line treatment of patients with EOC, we determined the expression of $\mathrm{p}-\mathrm{RB}$ protein in EOC by immunohistochemistry and analyzed its association with the clinicopathological features and the efficacy of CPT-11 chemotherapy.

\section{Methods}

\section{Ethics statement}

This study was reviewed and approved by the Ethics Committee of The Second Affiliated Hospital of Dalian Medical University (No. 2018064). Informed consent was obtained from each patient.

\section{Patient selection and clinical data}

From January 2002 to September 2016, 27 patients with recurrent ovarian cancer who underwent CPT-11 chemotherapy after first-line treatment failure at The Second Affiliated Hospital of Dalian Medical University were enrolled in this study. All patients were histologically confirmed as having EOC. These patients previously received ovarian cancer staging or cytoreductive surgery and completed adjuvant and/or neoadjuvant chemotherapy with cisplatin or carboplatin for 3-6 cycles. The patients were 41-74 years old, with a median age of 56 years old. Among them, 16 cases were poorly differentiated carcinoma, 7 cases were moderate-poorly differentiated carcinoma, and 4 cases were moderately differentiated carcinoma. According to the 2009 Ovarian Cancer Staging Criteria of the Federation of Gynecology and Obstetrics (FIGO), there were 4, 3, 18, and 2 patients with cancer stages I, II, III, and IV, respectively.

\section{Case entry criteria}

Inclusion criteria for this study were as follows: patient medical history information (including preoperative examination data, surgical pathology data, and follow-up information) was detailed and complete; the patients had pathologically confirmed EOC after staging determination or cytoreductive surgery; the patients received platinumcontaining regimens of 3-6 cycles; tumor recurrence was confirmed by computed tomography or magnetic 
resonance imaging; the patients received CPT-11-based regimen chemotherapy for no less than 2 cycles after firstline treatment failure; the patients had measurable tumor lesions according to RECST1.1 standard; and the Eastern Cooperative Oncology Group score was no less than 1 point. The exclusion criteria were as follows: the patients had ovarian lymphoma, metastatic ovarian cancer, ovarian germ cell tumors, or ovarian cord interstitial tumors; the patients had a history of other malignant tumors; the patients were lost during follow-up; the patients had no ovarian cancer staging or ovarian cancer cytoreductive surgery; the patients had second-line CPT-11-based chemotherapy for less than 2 cycles or did not receive the efficacy evaluation; and the patients had brain or epidural metastases.

\section{Patient follow-up and clinical characterization data}

The follow-up period was from the date of the first use of chemotherapy with CPT-11 until disease progression, death, or the follow-up deadline. The deadline for the follow-up was January 31, 2017. The follow-up contained patient treatment as well as survival and disease progression assessment, including but not limited to imaging examination, biochemical indicators, blood CA125 value, PFS, time of death, and the cause of death. If a patient was lost, the missing person was censored as of the date of loss. The time of death was the last follow-up time.

PFS was the time from the first day a patient used chemotherapy with the CPT-11 regimen until the tumor progressed or the patient died. The survival time after CPT-11 treatment was the time from the first day that the CPT-11-containing regimen was used until the day of death or the last follow-up date. The PFS time was calculated in days, and the CPT-11 survival time was calculated in months.

Platinum sensitivity was defined as patients receiving platinum-based adjuvant chemotherapy after the first operation until tumor recurrence or progression for an interval greater than 6 months; recurrence of less than 6 months was defined as platinum resistance.

The curative effect was defined according to the RECST1.1 solid tumor efficacy evaluation criteria and the International Society of Gynecologic Oncology ovarian cancer efficacy evaluation criteria. Complete remission (CR) was defined as all tumor lesions disappeared and/or the blood CA125 level decreased to normal $(\leq 35 \mathrm{U} / \mathrm{mL})$ for at least 4 weeks. Partial remission (PR) was when the sum of the maximal diameters of the baseline lesions decreased by at least $30 \%$ and/or the blood CA125 decreased by $50 \%$ of the baseline value for at least 4 weeks. Stable disease (SD) meant that the sum of the maximal diameters of the baseline lesions decreased but did not reach PR or increased but did not reach progressive disease (PD). PD was classified as the sum of the maximal diameters of the baseline lesions increasing by at least $20 \%$ or new lesions appearing, or when the patient's blood CA125 rose by more than 2 times of the baseline value during the follow-up period. The disease control rate was the sum of CR, PR, and SD.

\section{Immunobistochemistry}

After removal from the human body, all surgical specimens were fixed with $10 \%$ neutral-buffered formaldehyde solution in phosphate-buffered saline (PBS) for $24 \mathrm{~h}$, dehydrated in ethanol, embedded in paraffin, and stored at the Pathology Center of The Second Affiliated Hospital of Dalian Medical University. A total of 27 control cases were randomly selected during the same period from ovarian cyst excisions of normal ovarian tissue. Immunohistochemical analysis was performed on 4- $\mu$ m-thick sections. Paraffinfixed tissue sections were deparaffinized twice with xylol for $15 \mathrm{~min}$ and rehydrated with graded alcohol. After blocking endogenous peroxidase with $3 \%$ hydrogen peroxide for $10 \mathrm{~min}$, the slides were subjected to antigen retrieval for $5 \mathrm{~min}$ in a pressure cooker using sodium citrate buffer (pH 6.0), containing $0.1 \mathrm{M}$ citric acid and $0.1 \mathrm{M}$ sodium citrate in distilled water. After cooling to room temperature, sections were washed twice in PBS. Nonspecific binding was blocked by incubating the sections with normal goat serum, and the slides were incubated with the rabbit antihuman p-RB-Ser780 antibody (ab173289, Abcam, USA) at a 1:50 dilution in PBS at $4{ }^{\circ} \mathrm{C}$ overnight. The next day, after washing 3 times with PBS, the sections were incubated with secondary antibody and hydrogen peroxide for $30 \mathrm{~min}$. Following repeated washing with PBS, the sections were visualized using the 3,3'-diaminobenzidine substrate buffer for 3-5 min. Tissue sections were counterstained with hematoxylin and dehydrated in an ascending series of ethanol solutions ( $85-100 \%)$. After xylol treatment, the sections were mounted. As a control, duplicate sections were stained without primary antibodies. Positive staining of $\mathrm{p}-\mathrm{RB}$ in the nucleus was indicated by clear brownishyellow immunoreactive particles observed under a light microscope. Five high-power fields (400x magnification) were randomly selected for each section. Semi-quantitative 

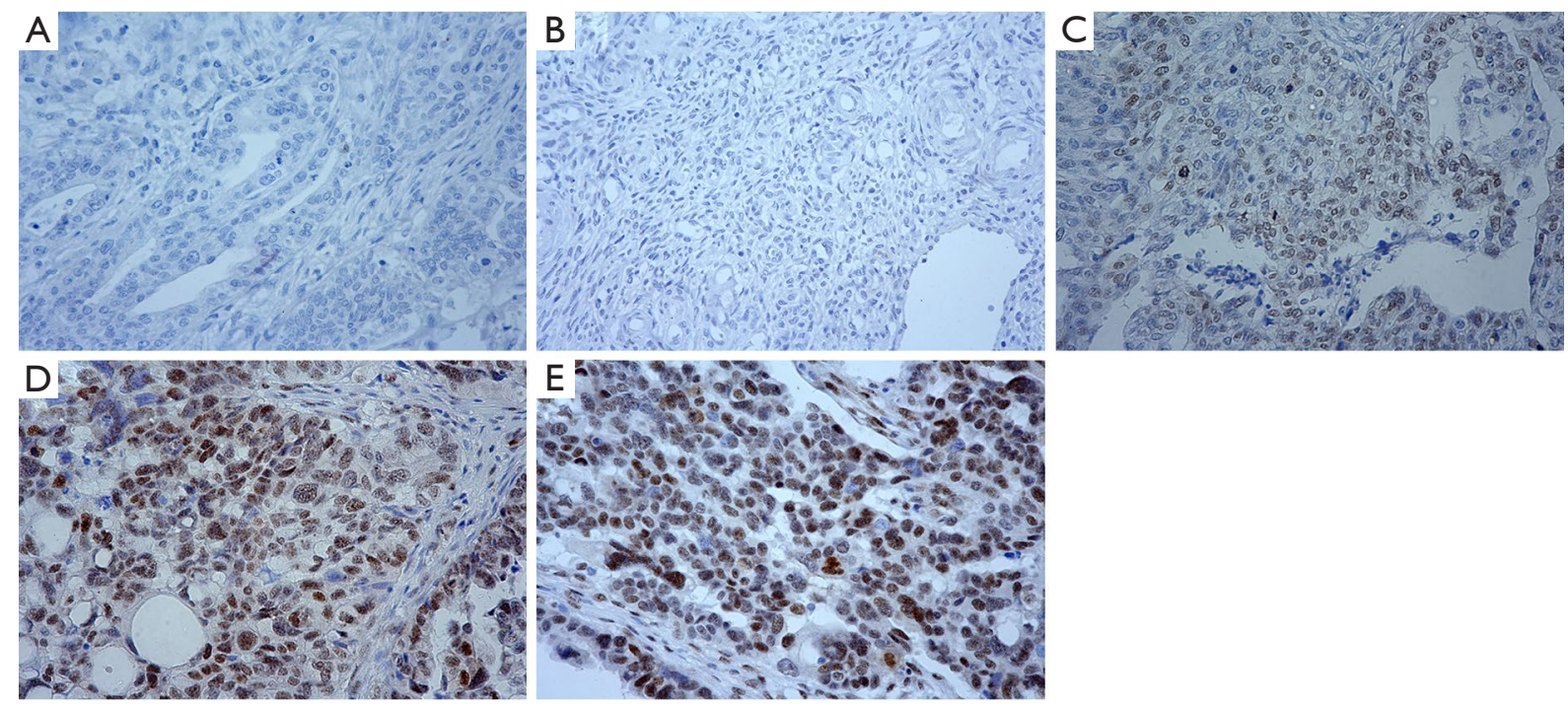

Figure 1 Representative p-RB staining images showing negative p-RB expression in normal tissues (A) and EOC tissues (B) along with weakly positive expression (C), positive expression (D), and strongly positive expression (E) of p-RB staining in EOC tissues. Magnification, 400×. p-RB, phosphorylated retinoblastoma; EOC, epithelial ovarian cancer.

evaluation results were obtained by combining the number of positive cells in the tissue sections with the percentage of the same type of cells observed and the staining intensity of the positive cells. According to the total number of counted cells and the percentage of positive cells, they were divided into 5 grades according to the percentage of positive cells: positive percentage of $0-5 \%, 0$ points; 6-25\%, 1 point; $26-50 \%, 2$ points; $51-75 \%, 3$ points; and $76-100 \%, 4$ points. Positive intensity was divided into 4 levels: no staining, 0 points; light brown-yellow staining, 1 point; brown-yellow staining, 2 points; and dark brownyellow staining, 3 points. The final score was the product of the positive cell percentage and the staining intensity grade (0-12 points). According to the score, it was divided into the following groups: $0-1$ point, negative (-); $2-4$ points, weakly positive $(+)$; $5-8$ points, moderately positive $(++)$; and 9-12 points, strongly positive (+++). All stained sections were independently analyzed by two pathologists, and the final immunohistochemical score was averaged.

\section{Statistical analysis}

SPSS17.0 software was used to analyze the data. The protein expression difference between groups was analyzed using the Chi-squared test or Fisher's exact test. The Cox proportional hazards regression model was performed for univariate and multivariate survival analyses. Multivariate analysis was evaluated by a Cox proportional hazards model. Overall survival was estimated by the Kaplan-Meier method and compared using the log-rank test. $\mathrm{P}<0.05$ for the difference was considered statistically significant.

\section{Results}

\section{Positive rate of $p-R B$ expression in EOC tissues was significantly higher than that in normal ovarian tissues}

Among the 27 normal ovarian tissues, immunohistochemical analysis determined that 6 tissues were positive and 21 tissues were negative for $\mathrm{p}-\mathrm{RB}$ expression (Figure 1A), with a positive rate of $22.22 \%$. Among the 27 cases of EOC, 17 cases were positive and 10 cases were negative for $\mathrm{p}-\mathrm{RB}$ expression (Figure 1B), with a positive rate of $62.96 \%$. Representative pictures of negative $\mathrm{p}-\mathrm{RB}$ expression in normal tissues and EOC tissues are shown in Figure 1A,B, respectively. Among the cases with p-RB-positive expression, weakly positive expression (Figure 1C) was found in 4 cases $(14.8 \%)$, positive expression (Figure 1D) was found in 7 cases (25.9\%), and strongly positive expression (Figure $1 E$ ) was found in 6 cases $(22.2 \%)$. The positive rate of $\mathrm{p}-\mathrm{RB}$ expression in the ovarian cancer patients was significantly higher than that in the normal ovarian tissues $\left(\chi^{2}=9.164, \mathrm{P}=0.002\right)$. The negative expression of $\mathrm{p}-\mathrm{RB}$ was classified as the $\mathrm{p}-\mathrm{RB}$ negative expression group (21 cases in 
Table 1 Phosphorylated retinoblastoma (p-RB) expression in normal ovarian and epithelial ovarian cancer tissues

\begin{tabular}{|c|c|c|c|c|c|c|c|c|}
\hline Group & Number of cases & \multicolumn{4}{|c|}{ p-RB expression } & Positive rate $(\%)$ & $\chi^{2}$ & $\mathrm{P}$ \\
\hline Normal ovarian tissues & 27 & 21 & 4 & 2 & 0 & 22.22 & 9.164 & 0.002 \\
\hline Epithelial ovarian cancer tissues & 27 & 10 & 4 & 7 & 6 & 62.96 & & \\
\hline
\end{tabular}

-, 0-1 point, negative; +, 2-4 points, weakly positive; ++, 5-8 points, moderately positive; +++, 9-12 points, strongly positive.

the normal group and 10 cases in the ovarian cancer group). The positive expression of $\mathrm{p}-\mathrm{RB}$ was classified as the $\mathrm{p}-\mathrm{RB}$ positive expression group ( 6 cases in the normal group and 17 cases in the ovarian cancer group) (Table 1).

\section{Relationship between $p-R B$ expression and the clinicopathological features of EOC patients}

Next, we analyzed the association of p-RB expression with the clinicopathological features of the EOC patients. There was no significant correlation between p-RB expression with the patient's age, FIGO stage, tumor differentiation, initial symptoms of ascites, tumor pathological type, platinum-sensitivity, lymph node metastasis, neoadjuvant chemotherapy, or the type of first-line chemotherapy (all $\mathrm{P}>0.05$, Table 2).

\section{$p-R B$ in epithelial ovarian tumors was significantly associated with disease control rates after CPT-11-based chemotherapy}

We evaluated the efficacy of chemotherapy in the 27 EOC patients according to the RECIST1.1 standard and the International Gynecologic Oncology Association evaluation criteria of ovarian cancer. Two cases (7.4\%) had the best response with CR and PR, 14 cases (51.9\%) were SD, and 11 cases $(40.7 \%)$ were PD. Therefore, the total disease control rate $(\mathrm{CR}+\mathrm{PR}+\mathrm{SD})$ was $59.3 \%$. The statistical results showed that $\mathrm{p}-\mathrm{RB}$ expression was significantly correlated with the control rate of CPT-11based chemotherapy in the EOC patients (Fisher's exact test, $\mathrm{P}<0.0001$, Table 3).

Univariate analysis showed that the median PFS of the recurrent patients with positive $\mathrm{p}-\mathrm{RB}$ expression after CPT11 chemotherapy was significantly higher than that of the patients with negative expression of p-RB (139 vs. 54 days, $\mathrm{P}=0.000)$. The median PFS after CPT-11 chemotherapy in patients with FIGO stage I-II disease was 215 days, which was significantly longer than that in patients with stage III-
IV disease ( 83 days, $\mathrm{P}=0.025)$. There was also a significant difference in the median PFS after CPT-11 chemotherapy in patients with initial symptoms of ascites (75 vs. 230 days, $\mathrm{P}=0.043)$. The median PFS was significantly shorter in patients with primary lymph node metastasis after CPT-11 chemotherapy than in those without lymph node metastasis (83 vs. 119 days, $\mathrm{P}=0.039$ ). There was no significant difference in the median PFS after chemotherapy with CPT-11 among the recurrent EOC patients in terms of age, tumor tissue differentiation, platinum-sensitivity, first-line chemotherapy, the line of neoadjuvant chemotherapy, or different pathological types (all $\mathrm{P}>0.05$, Figure 2 and Table 4).

\section{$p-R B$ expression is an independent prognostic factor for $P F S$ in the recurrent EOC patients who received CPT-11- based chemotherapy}

Cox regression model analysis demonstrated that $\mathrm{p}-\mathrm{RB}$ expression, FIGO stage, ascites, and lymph node metastasis were significantly associated with the median PFS after CPT-11-based chemotherapy in patients with recurrent EOC. However, only p-RB expression was an independent prognostic factor of PFS after application of CPT-11 in patients with ovarian cancer $(\mathrm{P}=0.026$, Table 5).

Univariate analysis showed that the survival time of patients with recurrent EOC after CPT-11 chemotherapy was not significantly associated with $\mathrm{p}-\mathrm{RB}$ expression, age, FIGO stage, tumor tissue differentiation, initial symptoms of ascites, lymph node metastasis at the first operation, platinum sensitivity, neoadjuvant chemotherapy, pathological type, or the type of first-line chemotherapy regimen (all $\mathrm{P}>0.05$, Table 6).

\section{Discussion}

In the present study, we found that the positive rate of p-RB expression in EOC tissue was significantly higher than that in normal ovarian tissue, suggesting that it may be involved in the occurrence of EOC. In addition, for 
Table 2 Association of phosphorylated retinoblastoma (p-RB) expression with the clinicopathological features of epithelial ovarian cancer patients

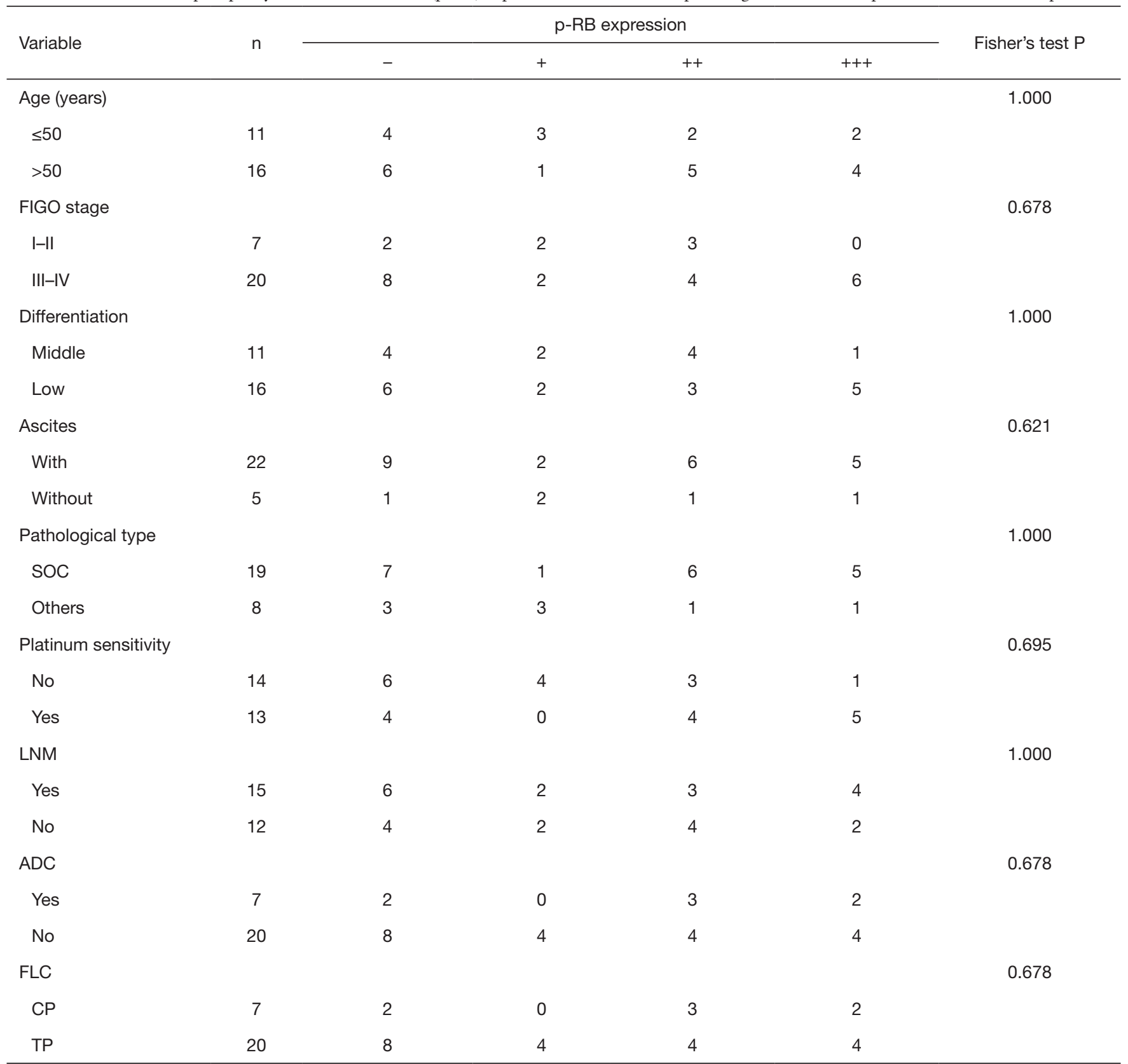

-, 0-1 point, negative; +, 2-4 points, weakly positive; ++, 5-8 points, moderately positive; +++, 9-12 points, strongly positive. FIGO, Federation of Gynecology and Obstetrics; SOC, serous ovarian cancer; LNM, lymph node metastasis; ADC, neoadjuvant chemotherapy; FLC, first-line chemotherapy.

patients with recurrent EOC, the expression level of p-RB was significantly related to the disease control rate and PFS following second-line CPT-11 treatment. Our findings suggest that $\mathrm{p}-\mathrm{RB}$ is a prognostic biomarker for recurrent EOC patients who accept CPT-11 therapy after first-line chemotherapy failure.

Ovarian cancer ranks first in female genital system cancer mortality. Due to the special anatomy of the ovary and the lack of specific methods for early detection, more than $80 \%$ of ovarian cancer patients are diagnosed at late stages (stage 
Table 3 Relationship between phosphorylated retinoblastoma (p-RB) expression and CPT-11-based chemotherapeutic efficacy in epithelial ovarian cancer

\begin{tabular}{lccc}
\hline \multirow{2}{*}{$\begin{array}{l}\text { p-RB } \\
\text { expression }\end{array}$} & \multicolumn{2}{c}{ Therapeutic efficacy (number of cases, \%) } & \multirow{2}{*}{$\begin{array}{c}\text { Fisher's } \\
\text { test P }\end{array}$} \\
\cline { 2 - 3 }- & CR/PR/SD & PD & $<0.0001$ \\
$+/++/+++$ & $1(10.0)$ & $9(90.0)$ & \\
\hline
\end{tabular}

,$- 0-1$ point, negative;,$+ 2-4$ points, weakly positive;,$++ 5-8$ points, moderately positive; +++, 9-12 points, strongly positive. $\mathrm{CR}$, complete remission; PR, partial remission; SD, stable disease; $\mathrm{PD}$, progressive disease.
III or IV) (2). Chemotherapy is currently one of the main treatments for ovarian cancer. However, in more than $70 \%$ of patients, there is recurrence due to platinum-based drug resistance. At the same time, the effective rate of secondline chemotherapy is only $9-40 \%$, and the 5 -year survival rate is less than $10 \%$ (11). Therefore, in order to improve the chemotherapeutic efficacy and overall survival after first-line chemotherapy failure, it is particularly important to discover predictors of second-line chemotherapeutic efficacy.
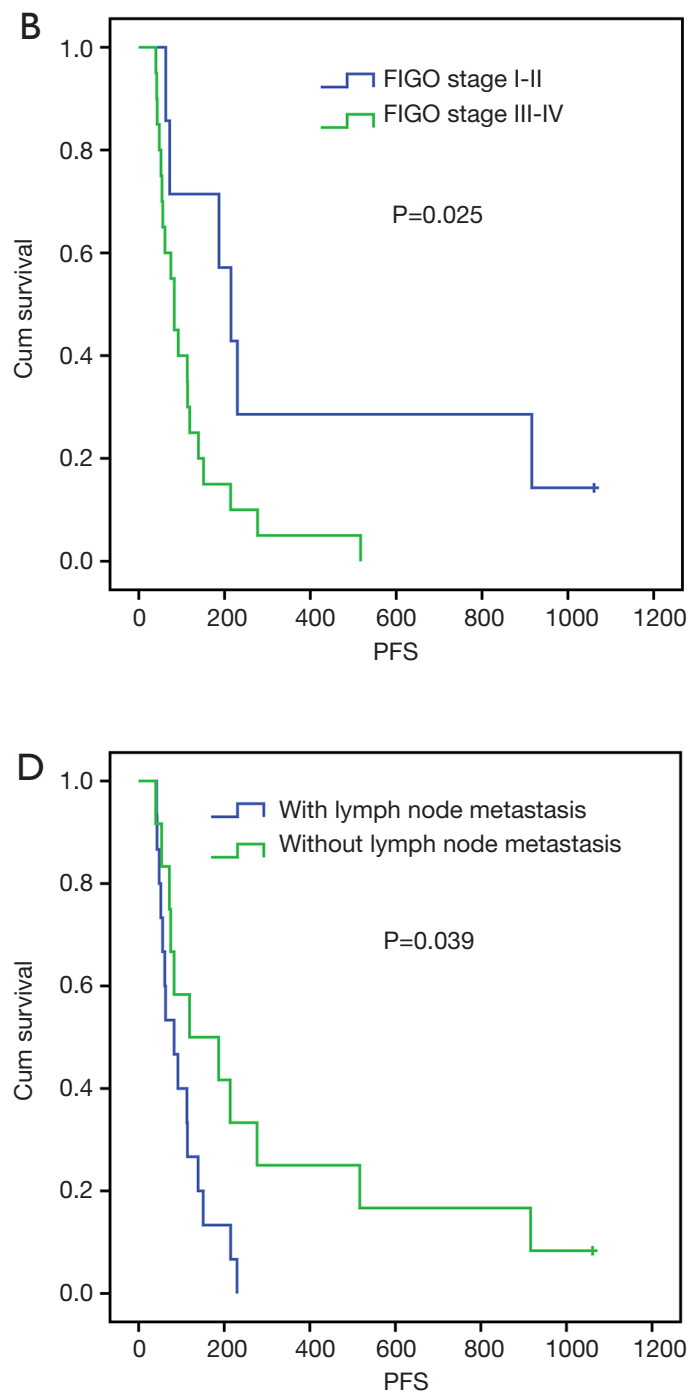

Figure 2 Multivariate analysis of PFS with phosphorylated retinoblastoma (p-RB) expression, Federation of Gynecology and Obstetrics (FIGO) stage, ascites, and lymph node metastasis of patients with recurrent EOC after CPT-11 chemotherapy. PFS, progression-free survival. 
Table 4 Univariate analysis of the median PFS after CPT-11 chemotherapy in patients with recurrent epithelial ovarian cancer

\begin{tabular}{|c|c|c|c|}
\hline Variable & Median PFS (days) & $\chi^{2}$ & $\mathrm{P}$ \\
\hline p-RB expression & & & 0.000 \\
\hline Negative & 54.0 & 15.315 & \\
\hline Positive & 139.0 & & \\
\hline Age (years) & & & 0.858 \\
\hline$\leq 50$ & 75.0 & 0.032 & \\
\hline$>50$ & 113.0 & & \\
\hline FIGO stage & & & 0.025 \\
\hline I-II & 215.0 & 5.058 & \\
\hline III-IV & 83.0 & & \\
\hline Differentiation & & & 0.863 \\
\hline Middle & 113.0 & 0.030 & \\
\hline Low & 83.0 & & \\
\hline Ascites & & & 0.043 \\
\hline With & 75.0 & 4.099 & \\
\hline Without & 230.0 & & \\
\hline Lymph node metastasis & & & 0.039 \\
\hline Yes & 83.0 & 4.240 & \\
\hline No & 119.0 & & \\
\hline Platinum sensitive & & & 0.459 \\
\hline Yes & 113.0 & 0.548 & \\
\hline No & 75.0 & & \\
\hline Neoadjuvant chemotherapy & & & 0.599 \\
\hline Yes & 139.0 & 0.276 & \\
\hline No & 75.0 & & \\
\hline Pathological type & & & 0.786 \\
\hline SOC & 114.0 & 0.074 & \\
\hline Other & 61.0 & & \\
\hline First-line chemotherapy & & & 0.850 \\
\hline $\mathrm{CP}$ & 63.0 & 0.036 & \\
\hline TP & 114.0 & & \\
\hline
\end{tabular}

PFS, progression-free survival; FIGO, Federation of Gynecology and Obstetrics; SOC, serous ovarian cancer; CP, cyclophosphamide combined with cis-platinum; TP, taxol combined with cis-platinum.
Topotecan is a recommended drug by the National Comprehensive Cancer Network guidelines for ovarian cancers that have failed first-line treatment, but its effect is often limited due to adverse effects such as intolerability, high cost, or lack of drug access. The efficacy of its analog, irinotecan hydrochloride (CPT-11), as a treatment for advanced ovarian cancer has been demonstrated both in basic and clinical trials (12) and is widely used. Currently, clinical treatment with CPT-11 as a second-line drug is usually based on the ovarian cancer pathological type or the uridine diphosphate glucuronyl transferase 1A1 (UGT1A1) gene polymorphism $(13,14)$. However, the UGT1A1 gene polymorphism has not been able to predict the efficacy of the index to develop chemotherapy regimens to achieve individualized treatment. Therefore, the identification of biomarkers for the curative effect of CPT-11 is of great significance to improve the curative effect of second-line and multi-line chemotherapeutics for ovarian cancer.

$\mathrm{RB}$ is a tumor suppressor and plays an important role in cell cycle progression (15). The phosphorylation status of RB changes dynamically with cell cycle progression (16-18). p-RB is elevated in colorectal cancer, breast cancer, and other solid tumors, suggesting a correlation of $\mathrm{p}-\mathrm{RB}$ with the development and progression of tumors. Zhang et al. (19) have found that $50.8 \%$ of breast cancer tissues had p-RB-positive expression. In addition, Liu et al. (20) have demonstrated that in normal breast epithelial cell lines, RB exists as a more active low-phosphorylated form. Meanwhile, in non-small cell lung cancer, osteosarcoma, human pharyngeal squamous carcinoma, and other tumor cell lines, RB protein was observed as the phosphorylated form (21). However, little is known about RB or p-RB in ovarian cancer. Seviour et al. (22) have analyzed the Cancer Genome Atlas (TCGA) database and found that ovarian cancer patients have a high p-RB signal. In our study, we detected $\mathrm{p}-\mathrm{RB}$ by immunohistochemistry in EOC patients and found that the positive rate of $\mathrm{p}-\mathrm{RB}$ protein in EOC $(62.96 \%)$ was significantly higher than that in normal ovarian tissue $(22.22 \%)$, suggesting that $\mathrm{p}-\mathrm{RB}$ plays an important role in the occurrence of EOC.

The relationship between $\mathrm{p}-\mathrm{RB}$ and clinicopathological features has been reported in different types of tumors. For example, $\mathrm{p}-\mathrm{RB}$ in breast cancer patients was significantly and positively correlated with the tumor size, clinical stage, and lymph node metastasis (19). Moreover, Xiao et al. (23) 
Table 5 Multivariate analysis of median PFS after chemotherapy with CPT-11 in patients with recurrent epithelial ovarian cancer

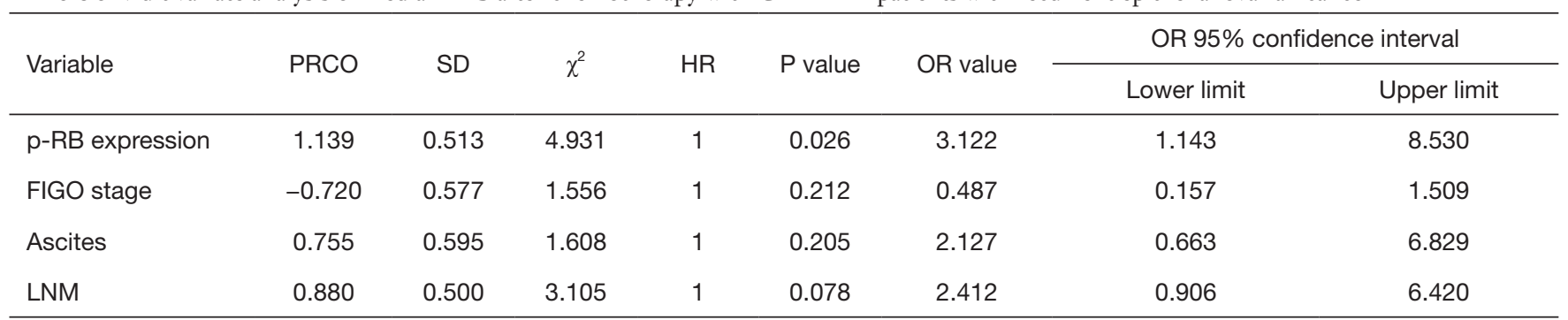

FIGO, Federation of Gynecology and Obstetrics; PRCO, partial regression coefficient; SD, standard deviation; HR, hazards ratio; OR, odds ratio; LNM, lymph node metastasis.

detected p-RB in 82 cases of thyroid papillary carcinoma by immunohistochemistry and observed a significant difference between $\mathrm{p}-\mathrm{RB}$ expression and the thyroid tumor size as well as lymph node metastasis. The results of this study showed that $\mathrm{p}-\mathrm{RB}$ was not statistically significantly associated with tumor differentiation, FIGO stage, lymph node metastasis, or other clinicopathological features. However, we found that $\mathrm{p}-\mathrm{RB}$ protein expression was significantly associated with the efficacy of CPT-11-based chemotherapy in the treatment of recurrent EOC. Our results were consistent with the observation that the response to topoisomerase 1 (TOP1) inhibitors correlates with tumor cell growth (24) and that $\mathrm{p}-\mathrm{RB}$ is correlated with a better CPT-11 response in colorectal cancer patients (10).

Progestin $\mathrm{X}$ receptor (25-28), multidrug resistance transporter ABCB1 (29), and ABCG2 (30) have been reported as predictive biomarkers related to the efficacy of CPT-11. Moreover, previous studies have shown that high levels of TOP1 expression are related to the efficacy of irinotecan (31), supporting the critical role for TOP1 in DNA replication (32). Furthermore, a large prospective study (UK MRC FOCUS trial) has shown that moderateto-high TOP1 expression is associated with a longer survival in patients with advanced colorectal cancer treated with irinotecan plus 5-fluorouracil (33). However, inconsistent results were obtained from the CAIRO study, showing that there was no correlation between TOP1 expression and the efficacy of irinotecan (34). In addition, another study has noted that cells acquiring resistance to SN-38 (the active metabolite of CPT-11) still maintained high levels of TOP1 expression (35). Therefore, the TOP1 expression level cannot be used to predict the clinical efficacy of CPT-11. In this study, the RB phosphorylation status not only predicted the rate of disease control in patients with recurrent ovarian cancer, but it also positively correlated with the PFS in patients receiving CPT-11. Our findings indicate that $\mathrm{p}-\mathrm{RB}$ expression is an independent prognostic factor affecting PFS after CPT-11 administration to patients with recurrent EOC.

Several studies have reported a relationship between p-RB expression and the survival of tumor patients. TCGA database analysis has found that a higher signal of $\mathrm{p}-\mathrm{RB}$ correlates with a poor survival in patients with ovarian cancer (18). Another study has demonstrated that $\mathrm{p}-\mathrm{RB}$ correlates with the survival of breast cancer patients (22). Recently, however, Ikai et al. (10) have discovered inconsistencies in colon cancer tissues. In our study, although a high expression of $\mathrm{p}-\mathrm{RB}$ protein was associated with a prolonged PFS in patients after CPT-11 chemotherapy, we did not find any improvement in survival after CPT-11 chemotherapy. Factors such as retrospective study design, follow-up treatment, sample size limitations, and inadequate follow-up may affect the end result. Therefore, additional studies with a large cohort of patient samples are needed to discover more specific proteins for predicting the therapeutic effect of CPT-11.

In conclusion, through this retrospective study, we found that the level of $\mathrm{p}-\mathrm{RB}$ protein might be a promising biomarker to predict the efficacy of CPT-11 chemotherapy in patients with recurrent ovarian cancer. Our findings will help to determine individualized and accurate treatments for EOC as well as stratification of EOC patients for CPT-11 chemotherapy after failure of first-line drug treatment. 
Table 6 Univariate analysis of survival time after CPT-11 chemotherapy in patients with recurrent epithelial ovarian cancer

\begin{tabular}{|c|c|c|c|}
\hline Variable & $\begin{array}{l}\text { Median survival } \\
\text { time (months) }\end{array}$ & $\chi^{2}$ & $P$ \\
\hline p-RB expression & & & 0.251 \\
\hline Positive & 18.7 & 1.316 & \\
\hline Negative & 58.6 & & \\
\hline Age (years) & & & 0.331 \\
\hline$\leq 50$ & 78.5 & 0.946 & \\
\hline$>50$ & 22.0 & & \\
\hline FIGO stage & & & 0.798 \\
\hline I-II & 33.5 & 0.066 & \\
\hline III-IV & 14.7 & & \\
\hline Differentiation & & & 0.621 \\
\hline Middle & 58.6 & 0.245 & \\
\hline Low & 33.5 & & \\
\hline Ascites & & & 0.932 \\
\hline With & 58.6 & 0.007 & \\
\hline Without & 19.2 & & \\
\hline Lymph node metastasis & & & 0.402 \\
\hline Yes & 22.2 & 0.701 & \\
\hline No & 58.6 & & \\
\hline Platinum sensitive & & & 0.206 \\
\hline Yes & 58.6 & 1.597 & \\
\hline No & 19.2 & & \\
\hline Neoadjuvant chemotherapy & & & 1.000 \\
\hline Yes & 33.5 & 0.000 & \\
\hline No & 22.2 & & \\
\hline Pathological type & & & 0.625 \\
\hline SOC & 33.5 & 0.238 & \\
\hline Others & 21.7 & & \\
\hline First-line chemotherapy & & & 0.352 \\
\hline $\mathrm{CP}$ & 35.7 & 0.865 & \\
\hline TP & 33.5 & & \\
\hline
\end{tabular}

FIGO, Federation of Gynecology and Obstetrics; SOC, serous ovarian cancer; $\mathrm{CP}$, cyclophosphamide combined with cis-platinum; TP, taxol combined with cis-platinum.

\section{Acknowledgments}

Funding: None.

\section{Footnote}

Conflicts of Interest: All authors have completed the ICMJE uniform disclosure form (available at http://dx.doi. org/10.21037/tcr.2018.12.19). The authors have no conflicts of interest to declare.

Ethical Statement: The authors are accountable for all aspects of the work in ensuring that questions related to the accuracy or integrity of any part of the work are appropriately investigated and resolved. The study was conducted in accordance with the Declaration of Helsinki (as revised in 2013). This study was reviewed and approved by the Ethics Committee of The Second Affiliated Hospital of Dalian Medical University (No. 2018064). Informed consent was obtained from each patient.

Open Access Statement: This is an Open Access article distributed in accordance with the Creative Commons Attribution-NonCommercial-NoDerivs 4.0 International License (CC BY-NC-ND 4.0), which permits the noncommercial replication and distribution of the article with the strict proviso that no changes or edits are made and the original work is properly cited (including links to both the formal publication through the relevant DOI and the license). See: https://creativecommons.org/licenses/by-nc-nd/4.0/.

\section{References}

1. Siegel RL, Miller KD, Jemal A. Cancer statistics, 2018. CA Cancer J Clin 2018;68:7-30.

2. Turkoglu O, Zeb A, Graham S, et al. Metabolomics of biomarker discovery in ovarian cancer: a systematic review of the current literature. Metabolomics 2016;12. doi: 10.1007/s11306-016-0990-0.

3. Hall M, Rustin G. Recurrent ovarian cancer: when and how to treat. Curr Oncol Rep 2011;13:459-71.

4. Ueda Y, Miyatake T, Nagamatsu M, et al. A phase II study of combination chemotherapy using docetaxel and irinotecan for TC-refractory or TC-resistant ovarian carcinomas (GOGO-OV2 study) and for primary clear or 
mucinous ovarian carcinomas (GOGO-OV3 Study). Eur J Obstet Gynecol Reprod Biol 2013;170:259-63.

5. Ichikawa R, Torii $\mathrm{Y}$, Oe S, et al. Retrospective comparative study of irinotecan and pegylated liposomal doxorubicin for platinum-resistant or -refractory epithelial ovarian and primary peritoneal carcinoma. Arch Gynecol Obstet 2014;290:979-84.

6. Hanker LC, Loibl S, Burchardi N, et al. The impact of second to sixth line therapy on survival of relapsed ovarian cancer after primary taxane/platinum-based therapy. Ann Oncol 2012;23:2605-12.

7. Mathijssen RH, van Alphen RJ, Verweij J, et al. Clinical pharmacokinetics and metabolism of irinotecan (CPT-11). Clin Cancer Res 2001;7:2182-94.

8. Hanahan D, Weinberg R. Hallmarks of cancer: the next generation. Cell 2011;144:646-74.

9. Fischer M, Müller GA. Cell cycle transcription control: DREAM/MuvB and RB-E2F complexes. Crit Rev Biochem Mol Biol 2017;52:638-62.

10. Ikai A, Watanabe M, Sowa Y, et al. Phosphorylated retinoblastoma protein is a potential predictive marker of irinotecan efficacy for colorectal cancer. Int J Oncol 2016;48:1297-304.

11. Siegel R, Naishadham D, Jemal A. Cancer statistics, 2013. CA Cancer J Clin 2013;63:11-30.

12. Sugiyama T, Yakushiji M, Ochiai K, et al. Japanese ovarian trials: focus on irinotecan. Oncology (Williston Park) 2003;17:29-33.

13. Xu Q, Ding YY, Song LX, et al. Correlation of UGT1A1 and ERCC1 gene polymorphisms with the outcome of combined irinotecan plus cisplatin treatment in recurrent ovarian cancer. Genet Mol Res 2015;14:7241-7.

14. Yoshihama T, Hirasawa A, Nomura H, et al. UGT1A1 polymorphism as a prognostic indicator of stage I ovarian clear cell carcinoma patients treated with irinotecan. Jpn J Clin Oncol 2017;47:170-4.

15. Ioachim EE, Malamou-Mitsi V, Kamina S, et al. Immunohistochemical expression of $\mathrm{Bcl}-2$ protein in breast lesions: correlation with Bax, p53, Rb, C-erbB-2, EGFR and proliferation indices. Anticancer Res 2000;20:4221-5.

16. Dong Y, Sui L, Sugimoto K, et al. Cyclin D1-CDK4 complex, a possible critical factor for cell proliferation and prognosis in laryngeal squamous cell carcinomas. Int J Cancer 2001;95:209-15.

17. Marsh KL, Varley JM. Frequent alterations of cell cycle regulators in early-stage breast lesions as detected by immunohistochemistry. Br J Cancer 1998;77:1460-8.
18. Sartor M, Steingrimsdottir H, Elamin F, et al. Role of p16/ MTS1, cyclin D1 and RB in primary oral cancer and oral cancer cell lines. Br J Cancer 1999;80:79-86.

19. Zhang MW, Liu F, Gu LN. Expressions of Rb and P-Rb in breast cancer tissues and their significance in TGF- $\beta$ induced epithelial-mesenchymal transition in breast cancer cells. Chinese Journal of Cancer Biotherapy 2016;1:95-101.

20. Liu RH, Xie G, Xiao ZX, et al. Cellular localization of $\mathrm{Pin} 1$ regulates the phosphorylationstatus of $\mathrm{Rb}$. Journal of Sichuan University (Natural Science Edition) 2016;53:419-23.

21. Liu JM. Study on p16-Cyclin D1-CDK4/6-Rb pathway in non-small-cell lung cancer. Central South University, 2013.

22. Seviour EG, Sehgal V, Lu Y, et al. Functional proteomics identifies miRNAs to target a p27/Myc/phospho$\mathrm{Rb}$ signature in breast and ovarian cancer. Oncogene 2016;35:691-701.

23. Xiao XL, Wu WC, Li ZR, et al. Correlation of nuclearcytoplasmic transport of $\mathrm{pRB}$ in the invase front and lymph node metastasis in papillary thyroid carcinoma. The Journal of Practical Medicine 2016;32:1602-5.

24. Wang K, Shrestha R, Wyatt A, et al. A meta-analysis approach for characterizing pan-cancer mechanisms of drug sensitivity in cell lines. PLoS One 2014;9:e103050.

25. Shi ZX, Tang Y, Li K, et al. Effect of pregnane X receptor (PXR) expression on chemotherapeutic sensitivity to CPT11 in colon cancer cells. Journal of Chongqing Medical University 2011;36:1381-3.

26. Raynal C, Pascussi JM, Leguelinel G, et al. Pregnane $\mathrm{X}$ Receptor (PXR) expression in colorectal cancer cells restricts irinotecan chemosensitivity through enhanced SN-38 glucuronidation. Mol Cancer 2010;9:46.

27. Mensah-Osman EJ, Thomas DG, Tabb MM, et al. Expression levels and activation of a PXR variant are directly related to drug resistance in osteosarcoma cell lines. Cancer 2007;109:957-65.

28. Chen Y, Tang Y, Wang MT, et al. Human pregnane X receptor and resistance to chemotherapy in prostate cancer. Cancer Res 2007;67:10361-7.

29. Ghazaly E, Perry J, Kitromilidou C, et al. Development and validation of an ultra-high performance LC-MS/MS assay for intracellular SN-38 in human solid tumour cell lines: comparison with a validated HPLC-fluorescence method. J Chromatogr B Analyt Technol Biomed Life Sci 2014;969:213-8. 
30. Mo W, Zhang J. Human ABCG2: structure, function, and its role in multidrug resistance. Int J Biochem Mol Biol 2012;3:1-27.

31. Gilbert DC, Chalmers AJ, El-Khamisy SF. Topoisomerase I inhibition in colorectal cancer: biomarkers and therapeutic targets. Br J Cancer 2012;106:18.

32. Hsiang YH, Liu LF. Identification of mammalian DNA topoisomerase I as an intracellular target of the anticancer drug camptothecin. Cancer Res 1988;48:1722-6.

33. Braun MS, Richman SD, Quirke P, et al. Predictive biomarkers of chemotherapy efficacy in colorectal cancer:

Cite this article as: Jiang K, Gao G, Yao L. Phosphorylated retinoblastoma is a biomarker of overall disease control and better progression-free survival of recurrent epithelial ovarian cancer patients treated with second-line topoisomerase inhibitor CPT-11 chemotherapy. Transl Cancer Res 2018;7(6):16821693. doi: 10.21037/tcr.2018.12.19 results from the UK MRC FOCUS trial. J Clin Oncol 2008;26:2690-8.

34. Koopman M, Antonini N, Douma J, et al. Sequential versus combination chemotherapy with capecitabine, irinotecan, and oxaliplatin in advanced colorectal cancer (CAIRO): a phase III randomised controlled trial. Lancet 2007;370:135-42.

35. Gongora C, Candeil L, Vezzio N, et al. Altered expression of cell proliferation-related and interferon-stimulated genes in colon cancer cells resistant to SN38. Cancer Biol Ther 2008;7:822-32. 